BULLETIN Bulletin hispanique

HISPANIQUE Université Michel de Montaigne Bordeaux

$116-2$ | 2014

Référentialité/autoréférentialité dans le roman espagnol contemporain : bilan et perspectives

\title{
Le roman-essai de Javier Marías
}

\section{Geneviève Champeau}

\section{(2) OpenEdition}

1 Journals

Édition électronique

URL : http://journals.openedition.org/bulletinhispanique/3346

DOI : 10.4000/bulletinhispanique.3346

ISBN : 979-10-300-0156-3

ISSN : $1775-3821$

Éditeur

Presses universitaires de Bordeaux

Édition imprimée

Date de publication : 1 décembre 2014

Pagination : 523-535

ISBN : 978-2-86781-963-6

ISSN : 0007-4640

\section{Référence électronique}

Geneviève Champeau, « Le roman-essai de Javier Marías », Bulletin hispanique [En ligne], 116-2 | 2014, mis en ligne le 01 décembre 2017, consulté le 09 mai 2019. URL : http://journals.openedition.org/ bulletinhispanique/3346; DOI : 10.4000/bulletinhispanique.3346 


\title{
Le roman-essai de Javier Marías
}

\author{
Geneviève Champeau \\ Université de Bordeaux Montaigne
}

Negra espalda del tiempo de Javier Marías et sa trilogie Tu rostro mañana subordonnent la métatextualité à une dimension cognitive de la littérature dans une modalité de la fiction, le roman-essai, mettant en scène une pensée narrative qui, par les voies de la digression, du commentaire et du métarécit, construit une interprétation plausible tout en réfléchissant sur les usages littéraires et sociaux du langage.

Mots-clés : Javier Marías, roman, essai, commentaire, métatextualité.

En Negra espalda del tiempo de Javier Marías y su trilogía Tu rostro mañana, la metatextualidad está al servicio de una dimensión cognitiva de la literatura en una modalidad de la ficción, la novela-ensayo, que escenifica un pensamiento narrativo y, mediante la digresión, el comentario y el metarelato, elabora una interpretación plausible al tiempo que reflexiona sobre los usos literarios y sociales del lenguaje.

Palabras claves: Javier Marías, novela, ensayo, comentario, metatextualidad.

Negra espalda del tiempo by Javier Marias, and his Tu rostro mañana trilogy subordinates metatextuality to a cognitive dimension of literature in a mode of fiction. This mode, the "essay novel" develops a narrative thought, through digression, commentary and metanarrative, to construct a plausible interpretation, while reflecting on literary and social uses of language.

Keywords: Javier Marías, novel, essay, commentary, metatextuality.

$\mathrm{T}$ oute œuvre de fiction traite, à des degrés divers et selon des modalités qui lui sont propres, du monde et d'elle-même, bien que le langage ne rejoigne jamais son référent. Selon les contextes de réception et les individus, le regard du lecteur tend néanmoins à privilégier l'une ou l'autre de ces tensions, l'univers raconté ou le signifiant verbal, la fonction référentielle ou poétique du langage. L'ouvrage Référence et autoréférence dans le roman espagnol

Bulletin Hispanique, Tome 116, n 2 - décembre 2014 - p. 523-535. 
contemporain $^{1}$, issu d'une rencontre scientifique de 1992, envisageait leur articulation, à un moment où se développaient les études sur la réflexivité du récit qui ont bénéficié d'un intérêt soutenu jusqu'au seuil du nouveau siècle. Après une phase d'hésitation terminologique à laquelle l'ouvrage en question n’échappe pas, le préfixe -méta s'est imposé ("metaficción », "metanovela ») pour désigner des récits développant des stratégies réflexives et ce, au détriment d' " autoréférence " à propos de laquelle Federico Bravo a souligné qu'elle était un concept précaire ${ }^{2}$.

Trois autres inflexions au moins sont perceptibles. Dans les années quatrevingt-dix, les œuvres soumises à l'analyse étaient des romans clairement identifiés comme tels; depuis cette date proliferent des œuvres hybrides qui mettent en question les frontières génériques et la distinction entre fiction et non-fiction, ce qui n'est pas sans incidences sur la diction du réel. Parallèlement, la fonction cognitive de la littérature a bénéficié en Espagne, à partir des années quatrevingt-dix, d'un regain d'intérêt en raison du développement de ce que l'on a appelé, avec plus ou moins de bonheur « le roman de la mémoire » qui prétend contribuer à l'élaboration d'une mémoire collective de la guerre civile et du franquisme empêchée par la dictature puis le " pacte de l'oubli » de la Transition démocratique. On assiste à un engouement du public pour le roman historique et à une révision critique de l'histoire du vingtième siècle européen ${ }^{3}$, à un " retour du social " tandis que, parallèlement, la recherche universitaire s'interroge sur le témoignage, l'engagement littéraire, l'exemplaritét. On constate encore un infléchissement méthodologique : le paradigme linguistique est concurrencé - sans être écarté - par une ouverture plus nette des études littéraires sur les sciences humaines 5 . L'intérêt renouvelé pour la référentialité construite par le discours littéraire ainsi que les travaux récents sur la prose narrative espagnole des dernières décennies invitent à penser que la réflexivité du récit est une forme actuellement privilégiée de la diction du réel, à concevoir plus que jamais la métatextualité comme une façon de scénariser un discours sur le réel qui ne prétend pas présenter ce dernier dans une illusoire immédiateté et met au

1. Référence et autoréférence dans le roman espagnol contemporain, Geneviève Champeau (coord.), Maison des Pays Ibériques, Bordeaux, 1994.

2. Federico Bravo, "Fiction, référence et signifiance " in Référence et autoréférence dans le roman espagnol contemporain, op. cit, p. 13-16.

3. Dans Sefarad (2001) de Antonio Muñoz Molina par exemple.

4. Cf. L'engagement littéraire, sous la direction d'Emmanuel Bouju, Presses Universitaires de Rennes, Cahiers du groupe $\varphi$, 2005 ; Témoignage et fiction dans l'Espagne contemporaine. Textes réunis par Jean-François Carcelen, Presses Universitaires de la Méditerranée, Montpellier, 2012 ; La ejemplaridad en la narrativa española contemporánea (1950-2010). Textos reunidos por Amélie Florenchie e Isabelle Touton, Iberoamericana/Vervuert, Madrid/Frankfurt, 2011 ; Isabelle Fauquet, L'exemplarité de la fiction dans le roman espagnol contemporain, thèse soutenue à l'université Michel de Montaigne - Bordeaux 3 en juillet 2012, consultable en ligne. URL : tel. archives-ouvertes.fr/docs.

5. Voir à ce sujet l'article de Franck Wagner «Le récit fictionnel et ses marges : état des lieux » in Vox poetica, revue en ligne, 15.IX. 2006 [consulté le 29 mai 2013]. disponible sur : http:// www.vox-poetica/t/wagner2006.html. 
contraire en représentation l'acte d'énonciation par lequel le sujet d'écriture s'efforce d'interpréter le monde ainsi que sa propre démarche intellectuelle et esthétique. En se signifiant elle-même, l'œuvre livre la façon dont elle signifie le monde et légitime son propre discours.

Les œuvres hybrides de Javier Marías, Negra espalda del tiempo (1998) et la trilogie Tu rostro mañana - dont je ne retiendrai dans cet article que le premier volume, Fiebre y lanza $(2002)^{6}$ car il prend pour objet le récit - sont un bon observatoire du rôle de la métatextualité dans la diction du réel à une époque qui a renoncé à l'idée de reproduction. Javier Marías affirme lui-même que " relatar lo ocurrido, dar cuenta de lo acaecido, dejar constancia de los hechos y delitos y hazañas es una mera ilusión o quimera" (NET, p. 10). Il est pourtant un de ces écrivains qui pratiquent le genre journalistique hybride de la columna où l'actualité est commentée dans une écriture qui emprunte aux recours de la fiction et de l'écriture littéraire. De même, sa pratique d'écriture dans NET et $F L$ relève d'une imbrication entre discours fictionnel et discours du savoir dans des hybrides qui ont été nommés « roman-essai » (J. C. Mainer) ou "novela pensamental» (G. Sobejano) $)^{7}$ et qui sont pour l'auteur une manière de "pensar sobre las cosas literariamente $"^{8}$. Une telle formule affiche la référentialité $\mathrm{du}$ récit, une dimension cognitive de la littérature, qui se manifeste dans la mise en scène de la pensée et la réflexivité de l'écriture. Sans doute ce dédoublement spéculaire n'est-il pas étranger à l'exacerbation du questionnement postmoderne des savoirs, du langage et de la représentation. Comment peut-on prétendre dire le monde quand les certitudes se sont écroulées et que les grands systèmes explicatifs n'ont plus cours?

\section{DigRESSION ET PENSÉE EN MOUVEMENT}

Dans les deux œuvres considérées, la réflexivité ne porte pas sur le genre roman, comme cela a été souvent le cas lorsque le roman, racontant l'histoire de sa propre élaboration, se fait work in progress. Elle transcende la généricité pour atteindre, en amont et de façon plus globale, le processus de la pensée quand elle adopte la forme narrative. Par de constantes digressions qui régissent l'économie du récit, celui-ci donne à voir, dans l'acte d'énonciation, comme cela se produit dans l'essai, la pensée saisie dans son surgissement.

L'intrigue est réduite à sa plus simple expression : incidence de la publication du roman précédent, Todas las almas', sur la vie de l'auteur (NET) et réflexion

6. Negra espalda del tiempo, Madrid, Alfaguara, 1998 et Tu rostro mañana. Vol. 1. Fiebre y lanza, Madrid, Alfaguara, 2002. Dorénavant, ces titres seront abrégés en NET et FL.

7. Gonzalo Sobejano, «Narrativa española 1950-2000: la novela, el género y las generaciones », in Arbor, n 693, sept. 2003 ; José Carlos Mainer, « Novela y ensayo » in La escritura desatada. El mundo de las novelas, Temas de hoy, Madrid, 2001, p. 161.

8. Propos de l'auteur reproduits dans Ángeles López, « Javier Marías : "La Academia no parece un lugar muy divertido" ", in La Clave, n 197, 21-27 janvier 2005, p. 79.

9. Javier Marías, Todas las almas, Barcelone, Anagrama, 1989. 
sur le récit autour de deux conversations de Jaime Deza et du professeur oxfordien Peter Wheeler $(F L)$. Minimale, elle n'est pas structurante puisqu' elle n'investit aucune des places décisives que sont l'incipit - consacré dans les deux œuvres à des réflexions du narrateur ${ }^{10}$ - et l'excipit - consacré, dans les deux cas également, à des récits métadiégétiques ${ }^{11}$. Sur la trame, reléguée au second plan, se greffe une prolifération d'histoires, de réflexions, d'images, de citations diverses qui s'enchaînent et s'entremêlent en excroissances multiples désignées dans le texte par les termes "rodeo", "digresión", "divagación" et " excurso». On peut légitimement étendre à ces œuvres ce que le professeur Wheeler applique à son art de la conversation qu'il définit comme "excurso de excurso de excurso" ( $F L$, p. 128). D'incessantes dérivations, parfois en cascade, ouvrent à chaque pas de nouvelles bifurcations dont le lecteur ne découvre que rétrospectivement quel lien elles entretiennent avec le fil thématique dont elles démultiplient les angles d'approche. Tours et détours qui ne deviennent entièrement signifiants que grâce à un système de reprises, parallèles, contrastes, échos de toutes sortes qui confèrent à ces deux ouvres un agencement musical, sur le modèle de la variation $^{12}$. C'est ainsi que le chapitre 11 de $N E T$, censé traiter de la mort de l'obscur écrivain anglais Wilfrid Ewart, le soir du 31 décembre 1922 à Mexico, reporte, de fait, la question aux chapitres 12 et 14 et substitue à l'information ainsi suspendue :

- le récit de l'enquête du narrateur pour se procurer l'information ;

- une critique des sources examinées (lettres, journaux, biographies, etc.) ;

- des considérations sur certaines œuvres de Javier Marías narrateur confondu avec l'auteur ;

- et sur différentes versions de l'histoire de Ewart que l'auteur Javier Marías a fait migrer, dans ses propres œuvres, en les reversant dans des textes de statuts différents : factuel (notice biographique) et fictionnel (nouvelle);

- s'y ajoutent des considérations sur sa pratique de l'apocryphe ;

- des réflexions d'ordre ontologique sur identité et récit ;

- et, enfin, une anecdote relative à Juan Benet, son mentor littéraire.

Les considérations sur l'écriture se mêlent à la démarche de l'écrivain, à la question des genres, à la relation entre narrateur et auteur, aux préférences littéraires de ce dernier et à l'articulation entre pratiques narratives et réflexion philosophique. Le passage met en abyme la structure digressive de l'œuvre entière, la pulsion centrifuge qui l'habite en chacun de ses points. À travers son " faire ", l'instance d'énonciation s'affiche au cœur de sa toile.

10. NET débute par des réflexions sur le narrateur et $F L$ sur les risques engendrés par la pratique du récit.

11. Ils seront abordés dans le troisième volet de cet article.

12. César Pérez García y voit l'influence de Tristram Shandy, traduit en espagnol par J. Marías, qu'il associe aux Variations Goldberg de Bach ("Sterne o el coloquio de los peldaños. En torno a Tu rostro mañana", in Alli donde uno diría que ya no puede haber nada. Tu rostro mañana de Javier Marías, Alexis Grohmann y Maarten Steenmeijer (eds.), Rodopi, Amsterdam - New York, 2009, p. 77. 
La syntaxe de la phrase fait écho à celle du récit. À la progression erratique de celui-ci correspond une hypertrophie de celle-là qui confronte le lecteur - en un rythme que l'espagnol nomme " moroso " - aux méandres d'une dérive hypotaxique multipliant incises et parenthèses - parfois enchâssées - ainsi qu'à une profusion de polysyndètes en " $y$ » et en " $o$ ", caractéristiques du style de cet auteur ${ }^{13}$. Le discours affine, nuance, rectifie et, qui plus est, ressasse par la récurrence de mots, d'énoncés, de motifs qui nourrissent sa progression tout en freinant celle de l'intrigue ou de ses détours. Le texte piétine, s'alimente de lui-même, en un mouvement à la fois progressif et régressif observé par Federico Bravo à propos de la prose de Juan Benet dont Javier Marías était l'ami et le disciple ${ }^{14}$. Cette actualisation du passé du texte dans son présent est la réalisation discursive de ce que Marías nomme "le dos noir du temps", autrement dit l'espace littéraire où ce qui a été ne cesse pas d'être.

Comme dans l'essai, l'élément unificateur de l'œuvre est ainsi dévolu non à l'intrigue ${ }^{15}$ mais à un thème, un objet culturel ${ }^{16}$ (réalité et fiction dans $N E T$, raconter dans $F L$ ) examiné sous des angles multiples, dans une démarche tâtonnante, progressant en spirale ${ }^{17}$, que Javier Marías définit luimême comme "errar con brújula " ${ }^{18}$. Le cheminement d'un " je " pensant, en quête d'un savoir sur lui et/ou sur le monde se manifeste d'ailleurs, dès l'image de couverture, dans le trait sinueux et discret d'un chemin (NET) et le ruban sombre de la route qui se déroule devant une moto qui laisse apparaître les mains du conducteur $(F L)$. L'ordre de la phrase et du récit a un enjeu épistémologique, il est la forme narrative d'une quête de savoir à une époque qui rejette la clôture d'une pensée doxologique ${ }^{19}$, qui inscrit la légitimité du discours dans un espace-temps donné tout en le rattachant à une subjectivité dont les marques sont partout présentes.

13. Elles ont été étudiées par Amélie Florenchie dans La répétition dans Negra espalda del tiempo de Javier Marías, Université Michel de Montaigne-Bordeaux 3, 2004 (URL:tel.archivesouvertes.fr/tel-00842119).

14. Federico Bravo, "L'écriture narrative de Juan Benet ou la syntaxe en spectacle ", in $\mathrm{La}$ licorne, $\mathrm{n}^{\circ} 31,1994$, p. 31-46.

15. "Nous avons posé - écrit Fernand Roy - que l'essai est un texte sans sujet, au sens où l'élément unificateur n’est jamais intégralement supporté par une intrigue, une histoire » ( Un tombeau littéraire pour l'essai ", in Études Littéraires, vol. 5, n 1, 1972, p. 26).

16. Cf. Gonzalo Sobejano, "Narrativa española 1950-2000: La novela, los géneros y las generaciones ", art. cit., p. 109.

17. José María Pozuelo Yvancos emploie l'expression « estructura en progreso " (in "Tu rostro mañana de Javier Marías : violencia, olvido y memoria ", in Alli donde uno diría que no puede haber nada, op. cit., p. 286).

18. Javier Marías, "Errar con brújula ", article figurant dans le recueil Literatura y fantasma, Madrid, Siruela, 1993, p. 91-93.

19. Cf. Pierre Glaudes et Jean-François Louette, L'essai, Paris, Hachette Supérieur, 1999, p. 48). Javier Marías se veut d'ailleurs l'héritier des Essais de Montaigne (FL, p. 450). 


\section{Commentaire et Pensée Discursive}

Si la structure du récit signifie par elle-même l'attitude réflexive du " Je " qui prend pour objet le mouvement de sa pensée, celle-ci est confortée par la nature du discours qui accorde le primat au commentaire, intimement mêlé à la narration, dans une diction du monde qui n'est pas étrangère à la " pensée discursive " caractéristique de l'essai ${ }^{20}$. Dans sa pratique de la conversation, le professeur Wheeler, père spirituel et alter ego du protagoniste Jaime Deza $(F L)$ fait l'éloge de l'« esprit spéculatif » qu'il oppose au pragmatisme anglais et qui est à ses yeux le sel de la conversation ("lo que a mí más me divierte » $F L$, p. 435-436). La pensée discursive est encore narrativisée lorsque le père de Deza l'incite à ne jamais se contenter d'une intellection spontanée ("Yqué más", nos decia después de que hubiéramos dado por concluidos, exhaustos, una exposición o un argumento " $F L$, p. 343), attitude à laquelle fait écho celle de Tupra, son chef dans la mystérieuse cellule d'espionnage ("Anda, sigue contándome qué más has visto ", $F L$, p. 116). Un scepticisme constructif, se démarquant de tout nihilisme (" nadie quiere saber nada », $F L$, p. 297) ${ }^{21}$, questionne sans relâche les savoirs constitués : "seguir pensando y seguir mirando más allá de lo necesario ", " más allá de la raya en la que uno se siente conforme [...] Alli donde uno diría que ya no puede haber nada» (FL, p. 344). Trois types de commentaires sont au service de cette quête d'intellection : le commentaire spéculatif, le commentaire-citation et le commentaire narratif.

\section{Le commentaire spéculatif}

Raconter et commenter sont les deux visages d'une écriture réflexive dans laquelle le narrateur se donne à voir voyant dans le va-et-vient entre récit et commentaire qui se motivent réciproquement. Le commentaire peut appeler le récit, comme c'est le cas dans les incipits, et le récit le commentaire dans le corps de l'œuvre. Les histoires racontées se prolongent dans - et sont interrompues par - des considérations généralisatrices énoncées au présent gnomique ${ }^{22}$ et à l'aide de pronoms incluant narrateur et narrataire (" uno ", " nosotros » et l'impersonnel "se ") en une démarche inductive qui dégage le général du particulier, la loi de l'expérience, dans des formules de ce type : "nunca hay nadie conforme del todo, ni con lo que elige " (NET, p. 49) ; "Quién sabe quién nos sustituye, sólo sabemos que se nos sustituye siempre» (FL, p. 47).

20. P. Glaudes et J.F. Louette, op. cit., p. 11.

21. Cela fait l'objet d'une longue tirade de Wheeler aux pages 297-298. Gonzalo Navajas souligne, pour sa part, que J. Marías « restablece y afirma la legitimidad y la vigencia de la escritura como un modo crítico y analítico del saber ", "Tu rostro mañana: teoría del saber de la narración ", in Allá donde uno diría que ya no puede haber nada. Tu rostro mañana de Javier Marías, op. cit., p. 149.

22. Weinrich souligne que les temps verbaux sont les marqueurs d'une attitude de locution et que le présent est celui du commentaire (Le temps, Paris, Seuil, 1973). 
Le rapport du narrateur personnage au savoir peut être mis en scène par le biais d'un récit second greffé sur le récit support et servant à son tour de support à la réflexion à la fois spéculative et spéculaire. C'est ce qui se produit dans l'épisode de la tache de sang que Deza découvre sur l'escalier de la maison du professeur Wheeler (FL, p. 166-183). Le récit premier met en scène le personnage narrateur confronté à une énigme qui déclenche un processus mental structuré par deux questions successives, l'une spéculative, l'autre pratique : quelle en est l'origine et que faire ${ }^{23}$ ? Leur traitement offre le spectacle d'une pensée analogique et déductive qui raisonne à partir d'exemples ancrés dans un contexte particulier : la question de l'origine de la tache fait naître le souvenir de Comendador, un ami d'enfance de Deza, trahi devant des douaniers par une goutte de sang tombée de son nez, indice d'une consommation de cocaïne. Puis la question "que faire ? " motive le déploiement du récit d'une mésaventure de Comendador qui, face à une jeune femme qui saigne et perd connaissance, craignant qu'elle ne soit morte, s'enfuit. Le narrateur-commentateur décline l'exemple sur différents registres : ontologique (existe-t-il un destin ?), éthique (de quoi sommes-nous capables ?) et esthétique (la fin colore téléologiquement un parcours vital dans les récits qu'en font les autres : c'est la motivation narrative - FL, p. 180 - ailleurs nommée " horror narrativo" - FL, p. 353). Les apparentes digressions enchaînées qui déploient le texte en éventail sont en réalité la manifestation d'une logique discursive paradigmatique plus que syntagmatique qui explore les déclinaisons d'un même motif sur différents registres. La réflexion n'est pas à proprement parler une argumentation logique, elle n'offre qu'un point de vue mais présente l'avantage de mettre à nu un processus mental dans son surgissement qui produit sur le lecteur le même effet d'authenticité que le monologue intérieur, rapprochant ainsi le commentaire réflexif du témoignage : l'ethos du sujet écrivant légitime la vérité énoncée.

\section{Le commentaire-citation}

Le commentaire prend aussi la forme de la citation récurrente dans laquelle le récit propre est glosé par le discours d'un autre. Si l'expérience est une des sources du savoir pour qui sait observer et déchiffrer, l'autre source est la voix des grands auteurs qui ont formulé une loi pouvant éclairer une nouvelle histoire, faire encore sens dans un nouveau contexte. Les titres Negra espalda del tiempo et Tu rostro mañana sont des citations de Shakespeare, tirées respectivement de Othello et de Henry IV ${ }^{24}$; Fiebre y lanza, de la tragédie The Indian Queen de Henry Purcell ${ }^{25}$. Le titre-citation motive le texte qui en est tout entier la glose.

23. José María Pozuelo Yvancos analyse la réthorique des « estados conjeturales » comme forme spécifique de la prose narrative de J. Marías dans son article « Tu rostro mañana de Javier Marías: violencia, olvido y memoria ", in Alli donde uno diría que ya no puede haber nada, op. cit., p. 295.

24. "What a disgrace it is to me to remember thy name! Or to know thy face tomorow ", Henry IV, $2^{\circ}$ partie, II, 2 (cf. Antonio Irriarte, "Citas y alusiones en Tu rostro mañana de Javier Marías ", ibidem, p. 316.

25. Cf. Antonio Irriarte, Ibid., p. 310-311. 
À l'intérieur du texte et entre parenthèses, les citations commentent le récit ou même un premier commentaire auquel elles sont juxtaposées. Deux citations jouissent de ce statut ${ }^{26}$. L'énoncé " "Apaga la luz, y luego apaga la luz" " (NET) est extrait d'Othello, au moment où celui-ci, sur le point de tuer Desdémone, a besoin de la répétition pour se convaincre lui-même. Dans $F L$, l'énoncé placé entre guillemets " ("Calla, calla y entonces sálvate") " pourrait bien être une pseudo-citation remplissant néanmoins le même rôle que la citation véritable et associée, dans sa première occurrence, à la «formule Miranda " par laquelle on déclare, aux États-Unis, à celui qui vient d'être arrêté : « tout ce que vous direz pourra être retenu contre vous ${ }^{27}$ (il est donc question des dangers du " cuento »). La récurrence de ces citations et les légères variations qui les altèrent dans leurs contextes successifs ${ }^{28}$ manifestent, par la seule variation du signifiant adapté à de nouveaux contextes, une tension paradoxale entre la relativité des vérités et la légitimité cognitive d'un discours littéraire transcendant l'expérience particulière.

\section{Le commentaire narratif}

Le récit premier fournit également des motifs spéculaires qui mettent en abyme le "faire " littéraire en rapport avec la visée cognitive. Ceux de la traduction et $\mathrm{du}$ rapport d'espionnage (" mis tareas de invención llamadas interpretaciones o llamadas informes ", FL, p. 316) sont à cet égard des manifestes littéraires narrativisés. L'auteur a lui-même développé une abondante activité de traducteur.

L'écriture se désigne en effet comme traduction, laquelle se décline en personnages traducteurs, en traduction effective de citations de toutes sortes (littéraires et extralittéraires) et en pseudo-traductions d'échanges épistolaires et de conversations (les deux conversations entre Deza et Wheeler, dans FL, sont censées s'être déroulées en anglais, le gros du roman étant de ce fait fictivement traduit). S'y ajoutent des commentaires métalinguistiques sur les ressources lexicales de différentes langues - le choix d'un terme, la difficulté d'en rendre la polysémie ("Podía traducirse y entenderse de varias maneras", " cabia toda una gama de interpretaciones menos literales ", FL, p. 167) qui montrent le traducteurécrivain aux prises avec un discours incertain, procédant par rectifications successives $^{29}$. Traduire/écrire n'est donc pas restituer mais interpréter.

26. NET, p. 143, 209, 220 ; FL ; p. 19, 183, 195, 201, 208, 460. Elles participent d'une poétique de la répétition/variation étudiée par Amélie Florenchie dans La répétition dans Negra espalda del tiempo de Javier Marías, op. cit.

27. "Cualquier cosa que diga podrá ser utilizada en contra suya » (FL, p. 17).

28. " "Apaga la luz, y luego apaga la luz" " (NET, p. 143 et 209) puis " "Apaga la luz, y luego apaga la luz", eso siempre ", (p. 220) : l'énoncé est repris sans guillemets ni parenthèses et intégré au texte dans la dernière phrase du livre : " hasta que la soñolienta mano de algún funcionario repara en el despilfarro y apaga la luz, y luego la apaga " (p. 404).

29. À preuve, ce commentaire sur la campagne consacrée au "careless talk ", à Londres, 
La synonymie entre traduction et interprétation est soulignée par le narrateur qui se nomme lui-même indifféremment "intérprete de personas " et " traductor [...] de vidas " (FL, p. 469). Sur le modèle herméneutique de l'enquête, il recherche des indices et établit entre eux des connexions jusqu'à conquérir un certain savoir narratif qui s'expose en un récit nommé informe dans le cadre de la cellule d'espionnage pour laquelle il travaille :

[...] escuchar y fijarme e interpretar y contar, [...] descifrar conductas, aptitudes, caracteres y escrúpulos, desapegos y convicciones, el egoismo, ambiciones, incondicionalidades, flaquezas, fuerzas, veracidades, repugnancias, indecisiones. Interpretaba - en tres palabras - historias, personas, vidas (FL, p. 262-263).

Polysémique, le terme informe est à prendre dans sa signification administrative et étymologique (du latin informare : "façonner, disposer, organiser ", mais également "tracer le portrait de quelqu'un "), étymologie qui le reverse dans le registre littéraire du portrait. Le questionnement générique de la biographie et de l'autobiographie (" dejar constancia de los hechos y delitos es una mera ilusión o quimera ", NET, p. 10) ${ }^{30}$ débouche sur la pratique d'un genre hybride ancien, la semblanza, auquel l'écrivain s'est exercé précédemment dans deux recueils, Vidas escritas (1992) et Miramientos (1997). Dans ces ouvrages, la semblanza, qui accompagne le portrait d'une personne d'une biographie succincte, est tirée vers la fiction humoristique et l'autofiction ${ }^{31}$ et revêt une tonalité ludique. Dans $N E T$, puis dans la variante du rapport des services secrets que propose $F L^{32}$, elle acquiert une visée pragmatique : connaître et prévoir pour agir ("deducibles historias no pasadas sino venideras, las que aún no habian ocurrido y podian por tanto remediarse ", FL, p. 32), l'informe étant déjà en lui-même surveillance et jugement (FL, p. 338).

Ces informes empruntent une voie spécifiquement littéraire de connaissance, celle de la fiction (ils sont qualifiés de "ejercicios [...] en fabulación ") qui subordonne les qualités analytico-déductives à l'intuition ("corazonada ",

pendant la seconde guerre mondiale : [...] " "careless talk, ¿has oído hablar de eso?” Me sonaba la expresión, "charla despreocupada" literalmente, o "negligente", o "descuidada", o "conversación imprudente", dificil una traducción satisfactoria y exacta, lo relacioné con lo que en español conocemos como "hablar a la ligera", aunque no sea tampoco eso, ni "cotilleo", ni "chismorreo", ni "habladurías" " (FL, p. 388).

30. Les entreprises biographique et autobiographique sont vaines pour de multiples raisons: sources insuffisantes, divergentes, voire invraisemblables et contradictoires, impuissance du langage à relater les faits, construction téléologique d'un récit qui forge artificiellement un destin, basculement de la biographie dans l'autofiction au gré des parallèles - voire des substitutions établis entre biographe et biographié.

31. Cf. Geneviève Champeau, "Retrato fotográfico, retrato literario y autoficción en Vidas escritas y Miramientos de Javier Marías ", in Relaciones transestéticas en la España contemporánea (Textes réunis par Geneviève Champeau, Bordeaux, Presses Universitaires de Bordeaux, 2011, p. 22-37).

32. FL en offre plusieurs exemples: Semblanza du militaire vénézuélien (p. 234-256), de la juge (p. 328-330), de l'acteur Dearlove (p. 350-365), de Deza lui-même (p. 346-347) dans le cadre de l'espionnage et, hors de celui-ci, de Tupra et de son amie (p. 63-72) ainsi que de l'attaché culturel De la Garza (p. 70-78). 
"fogonazo ", $F L$, p. 51, 382) d'une relation souterraine jusque-là inaperçue (" una asociación, un reconocimiento, una afinidad captada", FL, p. 382) ${ }^{33}$. Reposant sur une "intuición de la certeza $\|^{34}$, cette entreprise n'est pas sans danger, comme l'indique le lexique du risque (" aventura »/« aventurar », $F L$, p. 105, 320, 342), "arriesgar " (FL, p. 329, 340, 341, 342), "atrevimiento" (FL, p. 318, 338, 342), "osadia » (FL, p. 320, 340), « desparpajo» (FL, p. 338) et celui du pari ("apostar»/«apuesta», FL, p. 260, 263) 35. Dans un calcul des gains très pascalien ${ }^{36}$, Javier Marías parie sur un savoir de la fiction ("Puede saberse. Con equivocaciones, pero con muchos aciertos ", FL, p. 465), de l'ordre du plausible et du vraisemblable ${ }^{37}$, porté par une intime conviction (" se tiene una convicción, sin pasar por un solo argumento ", FL, p. 266), conviction que le narrateur insuffle à son destinataire, comme le fait Tupra, de toute évidence incarnation diégétique de l'instance énonciatrice dans la citation suivante :

Lo más notable era que no sólo conseguía que así sucediera a sus ojos, sino también a los de los demás, como si su visión se hiciera contagiosa cuando la relataba o, de otra manera, como si nos persuadiera y nos enseñara a ver lo que él veía al instante y nosotros no habríamos percibido nunca sin su concurso y sus descripciones y su indice que lo señalaba (FL, p. 64).

\section{MÉTARÉCIT ARCHÉTYPIQUE}

Au commentaire spéculatif, au commentaire-citation et au commentaire narratif qui diégétise la question de la référence s'ajoute un quatrième type de commentaire, que je nommerai métarécit archétypique. Métarécit n’est pas à prendre ici dans le sens de récit second porté par un récit premier que lui donne la narratologie ${ }^{38}$ (c'est, dans $N E T$, un récit second et dans $F L$ un récit premier), mais dans le sens où l'on parle de métalangage: un langage secondaire à propos d'un langage-objet. Il met en abyme, par une image développée en récit, l'œuvre qui le contient et dont il offre un commentaire condensé.

Chacune des deux œuvres est pourvue d'un tel métarécit : celui de l'homme et de la femme qui attendent le bus à l'aube, sous des lampadaires encore

33. Cf. la conversation sur l'horreur avec Alan Marriot ("los horrores dependen en buena medida de la asociación de ideas, habia dicho, "De la conjunción de ideas" ", FL, p. 378) et le rapprochement que réalise le narrateur entre la très jeune fille d'un couple d'amis et un vieil éditeur de revues sur la base d'une fugace expression identique sur leur visage ( $F L$, p. 381).

34. El ensayo español. Siglo XX, Jordi Gracia et Domingo Ródenas (eds.), Barcelone, Crítica, 2009, p. 964.

35. «[...] se trabaja sobre una base, aunque el principal punto de apoyo consiste siempre en una apuesta " (FL, p. 465). On lira à ce propos "La lección de Alan Marriot. Sobre los nexos nefastos y las parejas espantosas en Tu rostro mañana" de Rebeca Martín, in Alli donde uno diría que ya no puede haber nada, op. cit, p. 269-282.

36. Blaise Pascal, Pensées, fragment 397, édition de Michel Le Guern, Gallimard, Folio, 1977, vol. 2, p. 9-15.

37. "Al escuchar a la joven Nuix, me parece plausible cuanto ella advierte y me describe " (FL, p. 328).

38. Gérard Genette, Figures III, Paris, Seuil, p. 241-243. 
allumés $(N E T)$ et celui du narrateur qui se sent suivi dans la nuit $\left(F L^{39}\right.$. Dans le premier, le personnage narrateur Javier Marías observe deux personnages anonymes depuis sa fenêtre, imagine leurs pensées, ébauche une semblanza. Dans le second, Jaime Deza, le narrateur-protagoniste, raconte un épisode de sa propre vie londonienne. Les deux épisodes sont reliés à leur contexte proche par un lien analogique établi d'emblée par une comparaison entre les lampadaires allumés et une conception du temps sans coupures nettes dans $\mathrm{NET}^{40}$, entre l'interprétation des indices de l'aventure nocturne du narrateur protagoniste, et celle de la vie des autres, de jour, dans $F L: «[. .$.$] quien tiene por quehacer no$ fiarse acaba por percibirlo todo a esa luz suspicaz, recelosa, interpretativa " (FL, p. 318). Néanmoins, l'image filée (9 pages et deux photographies dans $N E T$, 13 pages dans $F L$ ) prolifère en un récit polysémique qui tisse à distance des liens multiples avec l'ensemble du réseau textuel, la ligne narrative devenant nœud.

L'image fournit un surplus d'intelligibilité en ramenant l'abstrait au sensible, pratique elle-même mise en abyme par un commentaire du narrateur sur l'art du récit de Tupra : "lograba que lo ilusorio adquiriera verbo y tomase cuerpo, y que se plasmase" (FL, p. 345). Si les informes sont qualifiés de figuraciones, c'est qu'ils marient les deux sens de figurar : "feindre ", "simuler " (c'est le propre de la fiction), ainsi que donner une forme sensible, incarner ce dont il est question.

Inversement, l'image-récit fait aussi voir/comprendre en un mouvement d'abstraction qui subsume des situations particulières en un récit archétypique, qui est à la fois une déclinaison supplémentaire des thèmes majeurs de l'œuvre et leur formulation synthétique. Elle participe conjointement d'un mouvement centrifuge (en un point du métarécit se nouent des liens analogiques avec divers constituants de l'œuvre) et, inversement, d'un mouvement centripète (le métarécit rassemble différents fils narratifs, axes sémantiques et procédures sémiotiques grâce à des récurrences de mots, d'images, des parallèles ou inversions, des échos de toutes sortes). La métaphore des lampadaires encore allumés à l'aube est ainsi le nœud d'un réseau sémantique complexe. Elle figure, d'une part, une conception du temps qui exclut les ruptures (dimension philosophique), concilie succession et simultanéité dans la coprésence des lumières de la nuit et de celles du jour (dimension existentielle). Cette coexistence des contraires subsume la diversité des hypothèses et des versions, les contradictions, les oxymores, les paradoxes égrenés au fil des digressions (par exemple, faire débuter un roman de 475 pages par "No debería uno contar nunca nada"), en une poétique de l'incertain. Le choix d'un moment de basculement (entre le jour et la nuit, entre deux âges, entre compagnie et abandon pour les personnages, entre condamnation et salvation) entre, par

39. NET p. 142-147, 281-282 et 401-404; FL p. 315-318, 367-373, 473-475.

40. «Todo es más misterioso, es más bien una prolongación artificial, atenuadora e inerte de lo que ha cesado y una resistencia protocolaria a ceder el paso o a señalar el inicio de lo que llega, como esas farolas que permanecen encendidas todavía un rato cuando ya ha amanecido en las grandes ciudades $[\ldots] »(N E T$, p. 142). 
ailleurs, en résonance avec toutes les situations de précarité (guerres, échecs, morts, trahisons, délations, abandons) récurrentes dans les histoires racontées (dimensions sociale et politique). Dans $F L$, la réversibilité de la situation (le protagoniste épié est lui-même un espion, la femme au chien qui suit Deza dans la nuit représente-t-elle un danger ou une opportunité ?) et l'oxymore du parapluie à la fois heaume et bouclier, lance et fusil $(F L$, p. 317) en font des variantes et des images archétypiques du couple confiance/défiance (dimension éthique), annoncé dès le début du livre, puis décliné à l'envi, et dont les deux modalités majeures et opposées sont la guerre (guerre civile, seconde guerre mondiale) et la conversation amicale.

Le métarécit signifie également par son emplacement. Constitué, dans les deux cas, de trois passages dont les deux premiers sont insérés dans le corps du texte et le troisième placé en position finale, il dénude son rapport au tout dont il est partie intégrante et qu'il éclaire rétrospectivement. En position conclusive, postposé à la fin de l'histoire, il fait rebondir celle du récit : "le mot de la fin " est une ouverture d'autant plus que, dans le métarécit, le bus emporte les deux personnages vers un futur incertain (NET) et qu'on ignorera jusqu'au volume suivant l'identité de la personne qui, après l'avoir suivi dans la nuit, frappe à la porte du narrateur $(F L)$. Le métarécit affiche de la sorte le refus de la clôture que ne cesse de proclamer la pensée en mouvement.

La prose de Javier Marías rejoint la pensée analogique dans cette " oscillation pour elle constitutive entre la ressemblance qu'elle signifie et la dissemblance qu'elle enjambe sans toutefois la réduire $»^{41}$, une ambiguïté inhérente au jeu des variations d'une écriture musicale. L'écriture analogique ne peut prouver quoi que ce soit, mais suggère, met sur la voie, peut "aider au passage " ${ }^{42}$. De même qu'en Droit, l'analogie est un procédé argumentatif dont on ne peut inférer que des conclusions probables ${ }^{43}$, le roman-essai de Javier Marías vise le plausible et maintient des zones d'ombre, d'incertitude, de mystère qui, pour lui, comme pour Juan Benet ${ }^{44}$, distinguent l'écriture littéraire de la pensée scientifique. La visée argumentative du roman-essai s'en trouve projetée au-delà des limites de la stricte rationalité pour atteindre la sphère des sensations et des affects comme le souligne J. A. Masoliver Ródenas, lorsqu’il évoque « [un] incomprensible susurro que nos persuade», "ese canto que queda siempre, más allá de su significado, como queda, vibrando, una lectura más allá de todos sus significados ${ }^{45}$.

41. Philibert Secrétan, L'analogie, Que sais-je ?, Paris, PUF, 1984, p. 7.

42. Jean Dieudonné, Analogie et connaissance, Collège de France, Séminaire interdisciplinaire, Paris, Malone, 1971, vol. 2, p. 168.

43. Manuel Atienza Rodríguez, Sobre la analogía en el Derecho. Ensayo de análisis de un razonamiento jurídico, Madrid, Cuadernos/Civitas, 1986, p. 34.

44. "[El] hombre de letras ha abandonado el único terreno donde el espiritu se siente seguro. Ese terreno sólo lo suministra la ciencia, aquella actividad que hace concordes el entorno con el pensamiento y que, por asi decirlo, se niega a tomar en consideración el poder de las tinieblas que rodean el área iluminada por el conocimiento" (Juan Benet, "Incertidumbre, memoria, fatalidad y temor ", in En ciernes, Madrid, Taurus, 1976, p. 61).

45. Juan Antonio Masoliver Ródenas, "Espejismos en una galería de espejos ", in Voces contemporáneas, Acantilado, Barcelone, 2004, p. 182. 


\section{UNE POÉTIQUE INTÉGRATRICE}

Dans une conception paradigmatique et polysémique du récit, la littérature n'est pas dissociée des autres champs du savoir. Le social et le politique, très présents, sont des aspects d'un discours surdéterminé qui se décline simultanément sur d'autres registres. L'écriture de Javier Marías diffère en cela profondément des réalismes espagnols du XXe siècle pour lesquels la fidélité au social tendait à être exclusive. En outre, la spécularité du récit, loin d'être le propre d'une "novela ensimismada " est la marque distinctive d'un sujet d'écriture qui, s'appréhendant dans l'altérité, ne dissocie pas la diction/fiction de soi de celle de la réalité extérieure, pas plus que la pensée spéculative de la création littéraire. Cette ouverture du champ de la fiction aux sciences humaines ébranle les distinctions génériques au bénéfice de récits hybrides tels que la semblanza qui oscille entre référentialité et fiction, et le roman-essai. La dénomination de l'œuvre s'en trouve affectée. Si NET a été qualifié par son auteur de "falsa novela ", FL ne porte aucun nom de baptême. En revanche, on relève au moins 106 occurrences de "contar »/ « cuento", 22 de "relatar »/ « relato" et 13 de " narrar", synonymes qui recouvrent une grande diversité de termes relatifs à divers aspects d'une activité perceptive, intellective et de communication : "preparar", "meditar", " maquinar ", " deformar ", "tergiversar ", " figurar ", "fabular "; " intuir ", " imaginar", " convencer "; " analizar ", " descifrar ", " traducir ", "interpretar", " desentrañar ", " desenmascarar "; " adivinar ", "ver ", "vislumbrar "; " opinar ", "dictaminar ", "diagnosticar", "pronosticar", "vaticinar". " Contar " déborde du champ littéraire ("eso no es una ficción, aunque si debe de ser un cuento ${ }^{46}$ ) pour devenir l'antonyme de "callar " et le synonyme de " hablar ". Il couvre toutes les facettes de l'échange humain, sans séparation entre usages sociaux et littéraires du langage, à l'écrit et à l'oral, dans le domaine de l'intime et du social, en synchronie ou en diachronie (les écrits sont des voix, le seul mode de conversation avec les morts, $F L$, p. 150, 241). Bienfait ou malédiction, don ou poison, le "cuento" fait exister : "las cosas no acaban de existir hasta que se las nombra" (FL, p. 14); "las figuraciones ya son hechos" (FL, p. 30). Il prolonge la vie des disparus dans les récits qu'ils laissent et ceux que les autres forgent à leur sujet ( $F L$, p. 352, 355-356, 361). ll est un mode d'existence, "mi manera de estar en el mundo" (FL, p. 27). L'élargissement se poursuit encore dans la dérivation à laquelle "contar " donne lieu : (" tener en cuenta ", "venir a cuento ", "darse cuenta", " contar con", " contar sin cuento", "pedir cuentas") qui, par le détour de l'étymologie (" computare», calculer et supputer), associe le récit à la collecte et à la sélection, au calcul, à la composition, à la prise de conscience ainsi qu'à l'évaluation car raconter, c'est finalement prendre position et donc prendre un risque.

46. Cet énoncé conclut l'épisode comique dans lequel est fictionnalisée la personne réelle de Francisco Rico. 